\title{
Recommendation from Peking Union Medical College Hospital for urgent hemodialysis during the COVID-19 pandemic
}

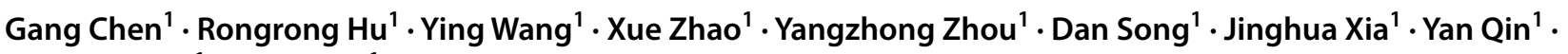 \\ Limeng Chen ${ }^{1} \cdot$ Xuemei $\mathrm{Li}^{1}$
}

Received: 19 May 2020 / Accepted: 1 June 2020 / Published online: 8 June 2020

○) Springer Nature B.V. 2020

Editor,

Until now, coronavirus disease 2019 (COVID-19) has resulted in more than 5 million confirmed cases, and the World Health Organization (WHO) has announced it as a global pandemic [1,2].

Infection prevention in the hemodialysis center of a general hospital is complicated by the monitoring of long-term dialysis patients, as well as the inevitable occurrence of emerging dialysis from wards or emergency room. Concordant with suggestions from the WHO, and based on real-world practice during the COVID-19 outbreak, we implemented a safe and efficient workflow to screen and manage patients who need urgent dialysis in Peking Union Medical College Hospital (PUMCH), a leading hospital in mainland China.

\section{Organizing the protection}

Based on environmental exposure degree, areas in PUMCH were classified as low risk, medium risk, high risk, and extremely high risk. The hemodialysis staff was required to wear the matched personal protective equipment (PPE) when a cross-area moving is needed. For example, the attending consultants should wear N95 masks, protective glasses, as well as suite up protective clothing and shoe covers, when they enter the extremely high-risk area such as the fever clinic. In addition, they should leave the contaminated PPE

Limeng Chen

chenlimeng@pumch.cn

Xuemei Li

lixmpumch@126.com

1 Department of Nephrology, Peking Union Medical College Hospital, Peking Union Medical College, Chinese Academy of Medical Science, Beijing 100730, China before they move back to lower risk zones. The staff maintains the same protective gear when they move towards areas with equal risks. Hand sanitizer was required whenever staff entered the hemodialysis center.

\section{Symptom screening and dialysis arrangement}

For the referred patients, we strictly analyze the indications for urgent dialysis. We recommended peritoneal dialysis and encourage catheter placement for patients with looming dialysis, but not urgent hemodialysis. For the inevitable urgent hemodialysis patients, we first collect their contact history, the temperature for the past 14 days, and potential suspicious symptoms and then implemented the corresponding process (Fig. 1).

We cooperate with the in-hospital Expert Group, which consists of specialists from the departments of infectious disease, respiratory, intensive care, and emergency medicine. We test SARS-CoV-2 swab for patients with suspicious contact, fever, respiratory symptoms, or chest imaging abnormalities. Patients with positive reading will be sent to the designated hospitals. We accept patients with negative swab readings but arrange separate dialysis during an observation period of 14 days. We also carefully monitor temperature for all contacted patients and evaluate the concerning symptoms daily, until all of them completely rule out the possibility of the infection.

During its development, the epidemiological features of COVID-19 are becoming blurred compared with its earlier onsets, and its clinical features show a certain degree of diversity $[3,4]$. For patients who need immediate dialysis, we will take them as potential COVID-19 patients and initiate continuous renal replacement therapy (CRRT) in situ when waiting for the swab results [5]. 
Fig. 1 Flowchart for the arrangement of patients needing urgent hemodialysis from wards or emergency room during the COVID-19 outbreak

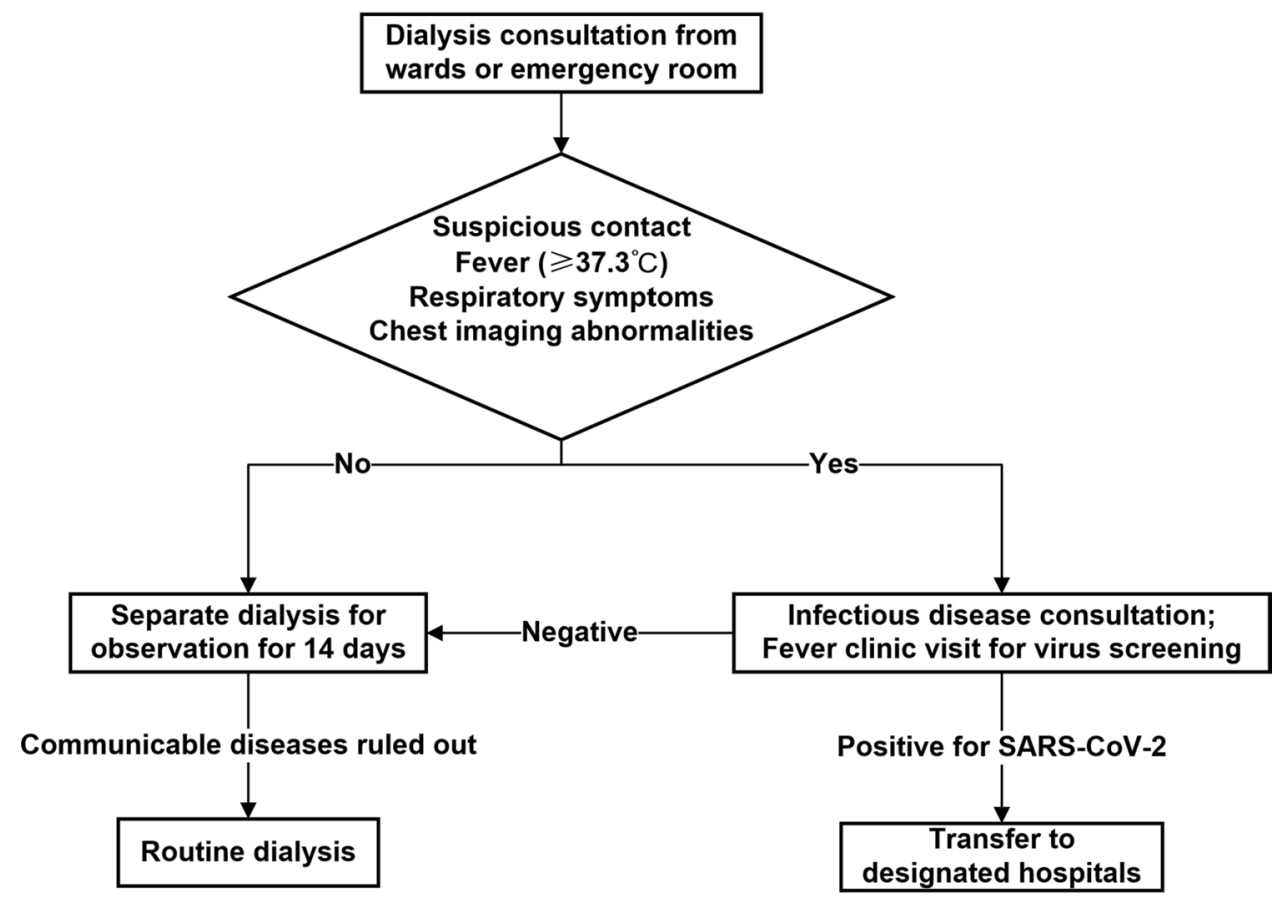

Staff who operate CRRT for uncleared patients should gear adequate PPE. The CRRT equipment should be disinfected after use [6]. We recommend chlorine-containing disinfectant to inactivate the possible pathogen effectively. We place the waste generated during CRRT in a double-packed biohazard bag and label it "infectious waste" for further processing [7]. The CRRT waste liquid is discharged according to the medical wastewater discharge standards [8].

Acknowledgements The work has been made available through an ISN-SRC Grant.

Author contributions GC generated the manuscript draft. RH, YW, XZ, YZ, DS, JX, YQ, LC, and XL reviewed and corrected the manuscript.

Funding This work was partly supported by the Key Research and Development Program of Ningxia Hui Autonomous Region (2018BFG02010 to C.L.), the Capital Specialized Clinical Application Project (Z171100001017196 to C.L.) and Chinese Academy of Medical Sciences Innovation Fund for Medical Sciences (CIFMS 2016-I2M-2-004 to C.L.).

Data availability We have presented all the necessary data in the manuscript. Related information is accessible upon requesting the corresponding author.

\section{Compliance with ethical standards}

Conflict of interest The authors declare that they have no competing interests.

\section{References}

1. Lai CC, Shih TP, Ko WC, Tang HJ, Hsueh PR (2020) Severe acute respiratory syndrome coronavirus 2 (SARS-CoV-2) and coronavirus disease-2019 (COVID-19): the epidemic and the challenges. Int J Antimicrob Agents 55:105924

2. Sohrabi C, Alsafi Z, O'Neill N, Khan M, Kerwan A, Al-Jabir A et al (2020) World Health Organization declares global emergency: a review of the 2019 novel coronavirus (COVID-19). Int J Surg (London, England) 76:71-76

3. National Health Commission of the People's Republic of China: Guidelines for novel coronavirus infection prevention and treatment (Trial 7th edition). https://www.chinalawtranslate.com/en/ coronavirus-treatment-plan-7/

4. Huang C, Wang Y, Li X, Ren L, Zhao J, Hu Y et al (2020) Clinical features of patients infected with 2019 novel coronavirus in Wuhan, China. Lancet 395:497-506

5. Expert team of Chinese Medical Association Nephrology Branch (2020) Recommendations for prevention and control of novel coronavirus infection in blood purification center (room) from the Chinese Medical Association Nephrology Branch (Trial Version 1). Chin J Nephrol 36:82-84

6. National Health Commission of the People's Republic of China: Regulation of disinfection technique in healthcare settings. http:// www.nhc.gov.cn/wjw/s9496/201204/54510/files/2c7560199b 9d42d7b4fce28eed1b7be0.PDF

7. State Council of People's Republic of China: Regulations on the Management of Medical Waste. http://www.gov.cn/gongbao/conte nt/2003/content_62236.htm

8. National Environmental Protection Administration \& National Administration of Quality Supervision, Inspection and Quarantine: Standards for the discharge of waters in medical institutions. China Environment Press. 2006; GB 18466-2005

Publisher's Note Springer Nature remains neutral with regard to jurisdictional claims in published maps and institutional affiliations. 